\title{
Vertebroplasty and kyphoplasty: techniques for avoiding complications and pitfalls
}

\author{
Wade Wong, D.O., F.A.C.R., AND John M. Mathis, M.D. \\ Interventional Neuroradiology Section, University of California, San Diego, California; \\ and Department of Radiology, Lewis Gale Medical Center, Salem, Virginia
}

The purpose of this article is to present a series of common complications and pitfalls associated with vertebroplasty and kyphoplasty, with discussions on how to avoid those problems in a practical, case-based essay.

\author{
KEY WORDS - vertebral compression fracture - vertebroplasty • kyphoplasty • \\ complication
}

Complication rates for vertebroplasty and kyphoplasty have been reported to range from less than $2 \%$ for osteoporotic VCFs to up to $10 \%$ for malignant tumor-related VCFs. ${ }^{2,3,5,8,9,11}$ The complications have included radiculopathy, paralysis, worsening pain, pulmonary emboli, bleeding, infection, and rib fractures. The potential for pneumothorax is always present whenever physicians work in the thoracic region. New VCFs adjacent to those that have already been treated have been implicated, particularly when large amounts of cement have leaked into the adjacent disc spaces. ${ }^{4}$ This supposition has been somewhat difficult to validate, however.

Many of the complications of vertebroplasty are directly related to cement leaks. In that regard, the manufacturers and promotors of kyphoplasty claim some advantage for this procedure over vertebroplasty, in that containment of thick, viscous material within a balloon-created cavity yields less cement leakage than forcing thin, runny, more liquid cement throughout the interstices of a fractured vertebra, as is done in vertebroplasty. ${ }^{7}$ Nevertheless, a cement leak does not necessarily mean a complication. Many experienced operators specializing in vertebroplasty have performed hundreds and even thousands of these procedures without ever creating a complication due to cement leakage. ${ }^{10}$ The real issue is visualization of the cement and control of its application. The following hypothetical cases represent examples of common pitfalls.

\section{HYPOTHETICAL CASES}

\section{Case 1}

A 44-year-old man with human immunodeficiency virus and a history of abdominal lymphoma presents with low-back pain. The referring internist requests that

\footnotetext{
Abbreviations used in this paper: $\mathrm{AP}=$ anteroposterior; $\mathrm{CT}=$ computerized tomography; $\mathrm{MR}=$ magnetic resonance; $\mathrm{VB}=$ vertebral body; $\mathrm{VCF}=$ vertebral compression fracture.
}

you perform vertebroplasty. In your physical examination you find pain at the L-3 and L-5 levels. (Figure 1A, an MR image obtained at admission, reveals destructive lesions of L-3 and L-5 consistent with a history of lymphoma.) When giving his medical history, however, the patient mentions frequent night sweats. How should you proceed?

Obviously, some patients, especially those who have human immunodeficiency virus, may have more than one malady. In a patient with a history of night sweats, concern about an underlying infection should set off alarms. A biopsy procedure (as demonstrated on the fluoroscopic image in Fig. 1B) prior to vertebroplasty would therefore be prudent.

In this patient, biopsy findings revealed the presence of tuberculosis in the vertebrae, despite a history of abdominal lymphoma. The presence of infection in the target vertebrae is considered a contraindication for performing a vertebroplasty or kyphoplasty.

\section{Case 2}

A 66-year-old man seeks medical care 1 week after a fall. He reports severe low-back pain on presentation. The admission MR image (Fig. 2A) demonstrates edema and compression of the L-5 VB.

Physical examination correlates the pain with the L-5 VCF. When the patient gives his medical history, however, he mentions having difficulty recently in emptying his bladder. How should you proceed?

A CT scan can be extremely helpful in delineating complex fractures, especially if there is a hint of cauda equina or the potential for instability. In this case, the CT scan (Fig. 2B) reveals a retropulsed fragment that could account for the development of cauda equina. In addition, fractures are seen in the posterior cortex of the L-5 vertebra. These could become a conduit for cement leaks into the spinal canal. This amount of retropulsion is clinically significant enough that neither a kyphoplasty nor a verte- 


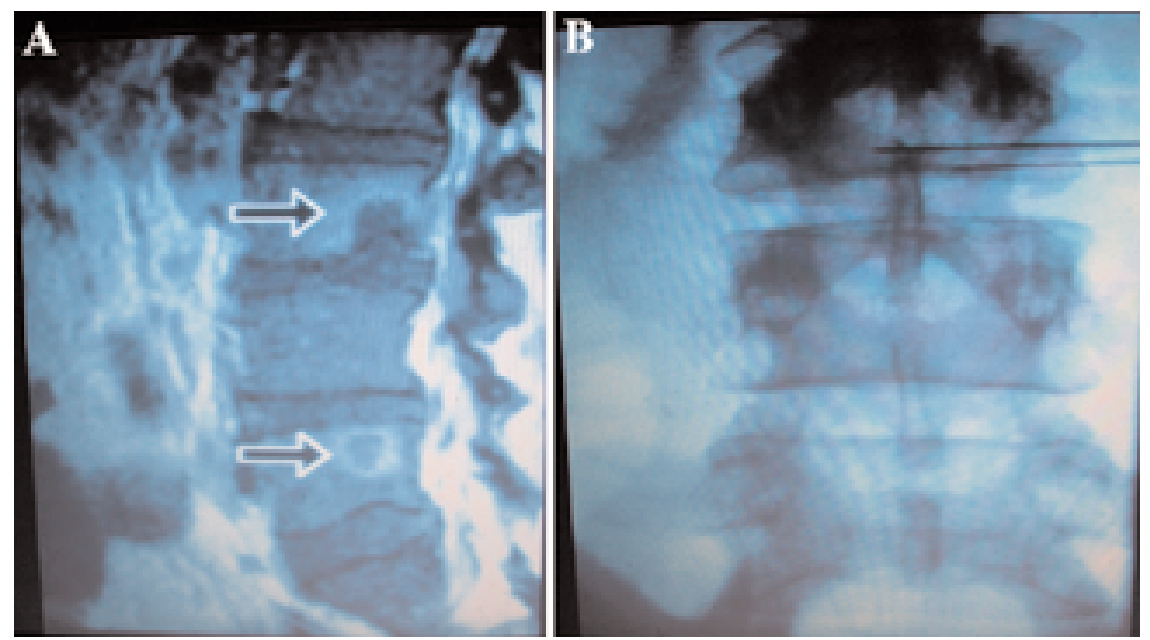

Fig. 1. A: Sagittal $\mathrm{T}_{1}$-weighted MR image of the lumbar spine. B: Anteroposterior fluoroscopic image obtained during biopsy.

broplasty would be indicated. This patient eventually underwent a spinal decompression procedure.

\section{Case 3}

A 78-year-old woman trips on the hospital carpet when she is being discharged. She immediately experiences excruciating mid-back pain. The hospital administrator demands that you promptly perform a kyphoplasty. Your examination with fluoroscopy (Fig. 3A) confirms that the patient is tender at T-8 and T-9, where compression fractures are observed. She is neurologically intact. You obtain MR images (Fig. 3B and C), which are somewhat confusing because there appears to be a piece of bone that is misaligned. How should you proceed?

For cortical bone detail, CT scans are more definitive than MR images. The CT scans obtained in this patient (Fig. 3D-I) reveal complex fractures that involve three columns of the T-8 and T-9 vertebrae. This is a highly unstable situation. This patient therefore needs a spinal decompressive and stabilizing surgical procedure instead of a kyphoplasty or vertebroplasty.
A good way to stay out of trouble when performing vertebroplasty or kyphoplasty is to avoid situations that are contraindicated. Table 1 presents a summary list of contraindications or guidelines that we recommend. This list of contraindications is based on the American College of Radiology Standards for the Performance of Vertebroplasty. ${ }^{6}$

\section{Case 4}

A 68-year-old woman undergoing a kyphoplasty. Figure $4 \mathrm{~A}$ is a fluoroscopic image obtained during the procedure. What happened and how could this have been avoided?

This procedure is being performed with the aid of a portable fluoroscope that is handled by an inexperienced technician. The surgeon becomes reluctant to request changes in visualization from the AP to the lateral plane when the technician almost drops the fluoroscope on the needle. As a result, the surgeon decides to advance the drill with the fluoroscopic view in the AP instead of the lateral plane. When he finally does request an image in the
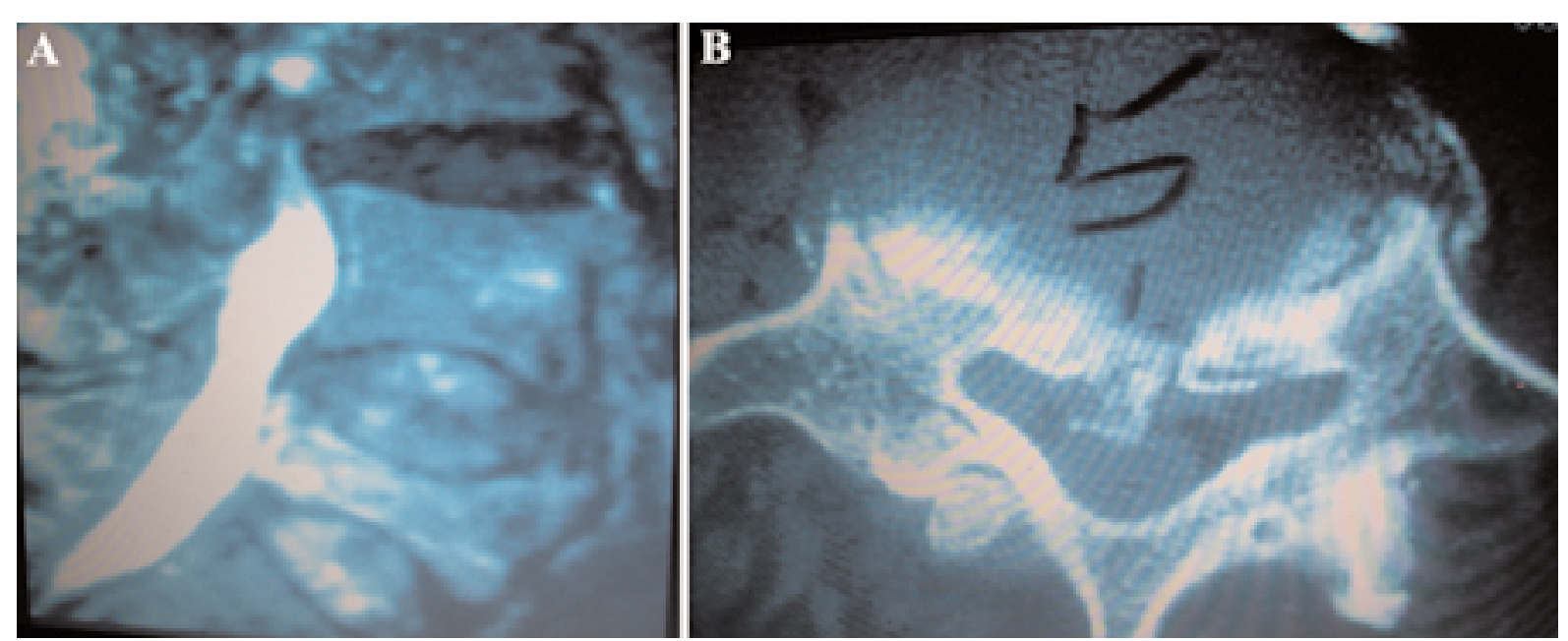

Fig. 2. A: Sagittal $T_{2}$-weighted MR image of the lumbar spine. B: Axial CT scan through L-5 of VCF. 


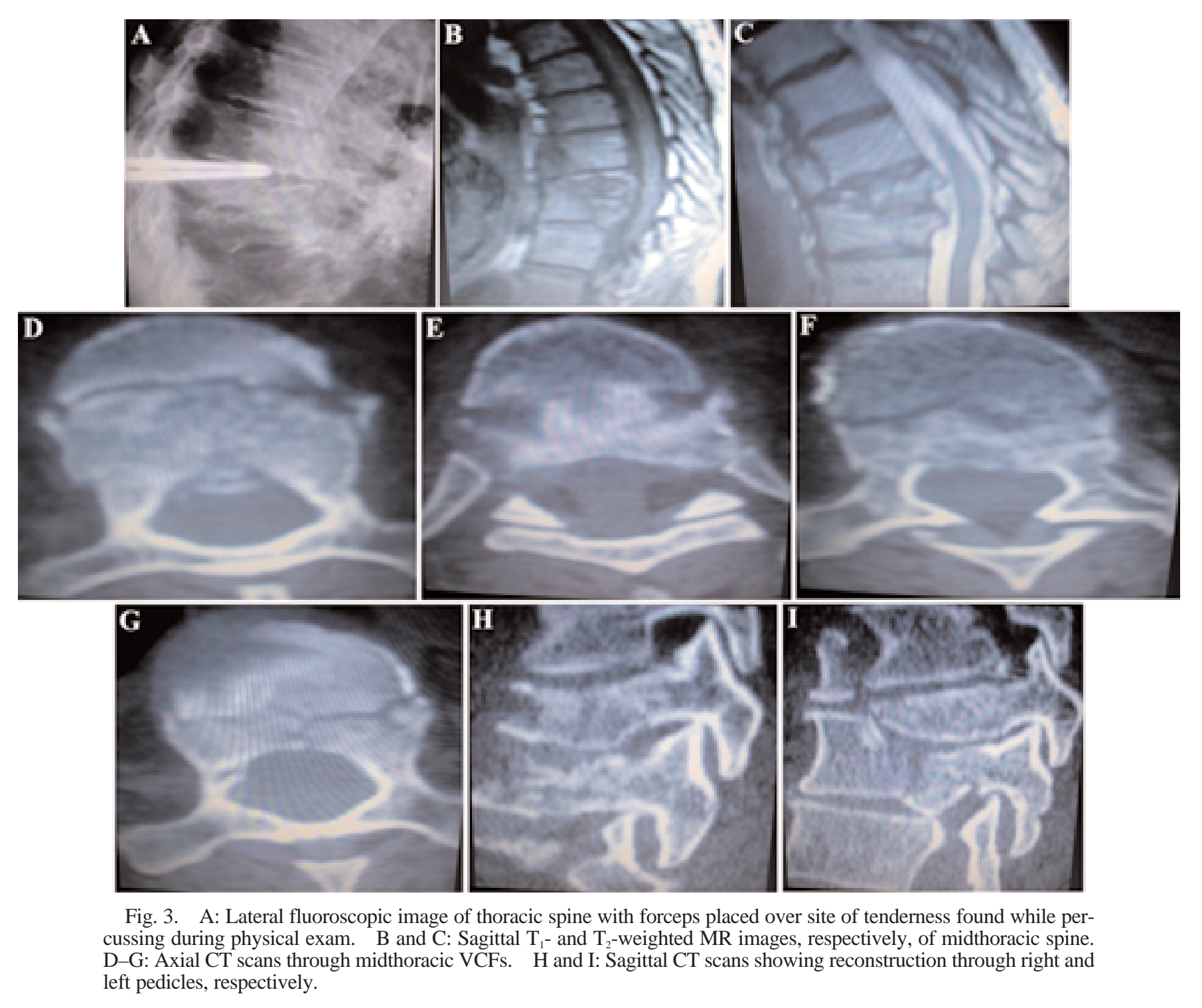

lateral plane, he discovers that the drill tip has migrated more than several centimeters anterior to the VB. Fortunately, there is no bad outcome, even though the potential for bowel perforation or vascular injury is extremely high. This reinforces the necessity of acquiring images in the lateral plane whenever one is advancing instruments such as needles or drills. Having biplane fluoroscopy available can expedite the process.

With single-plane fluoroscopy, establishing isocentricity can also be very helpful. Isocentricity is the ability to produce images in rapid rotation around the target vertebra, which allows multiple angles of visualization instantly without having to move the patient. To establish isocentricity, one must place the target (that is, the spine) in the center of the fluoroscopic image on the AP plane, then raise or lower the fluoroscopic table as necessary so that the target is centered on the lateral view. Isocentricity will allow rapid movement from lateral to oblique to AP views of the target without having to raise, lower, or move the table from side to side. Figure 4B-E consists of intraoperative photographs showing the apparatus in various positions.

Unfortunately, some portable C-arm fluoroscopes have arms too small to allow isocentricity. In those cases, having two $\mathrm{C}$ arms may help to enable at least biplane visualization rapidly. Vertebroplasty and kyphoplasty are imaging-guided procedures, so the operator should make use of every possible advantage.

\section{Case 5}

A 78-year-old woman undergoes vertebroplasty at L-3, which results in bilateral L-3 radiculopathies (Fig. 5). Why?

\section{Case 6}

A 70-year-old woman becomes extremely paretic in both lower extremities following T-12 vertebroplasty (Fig. 6A). Why? 


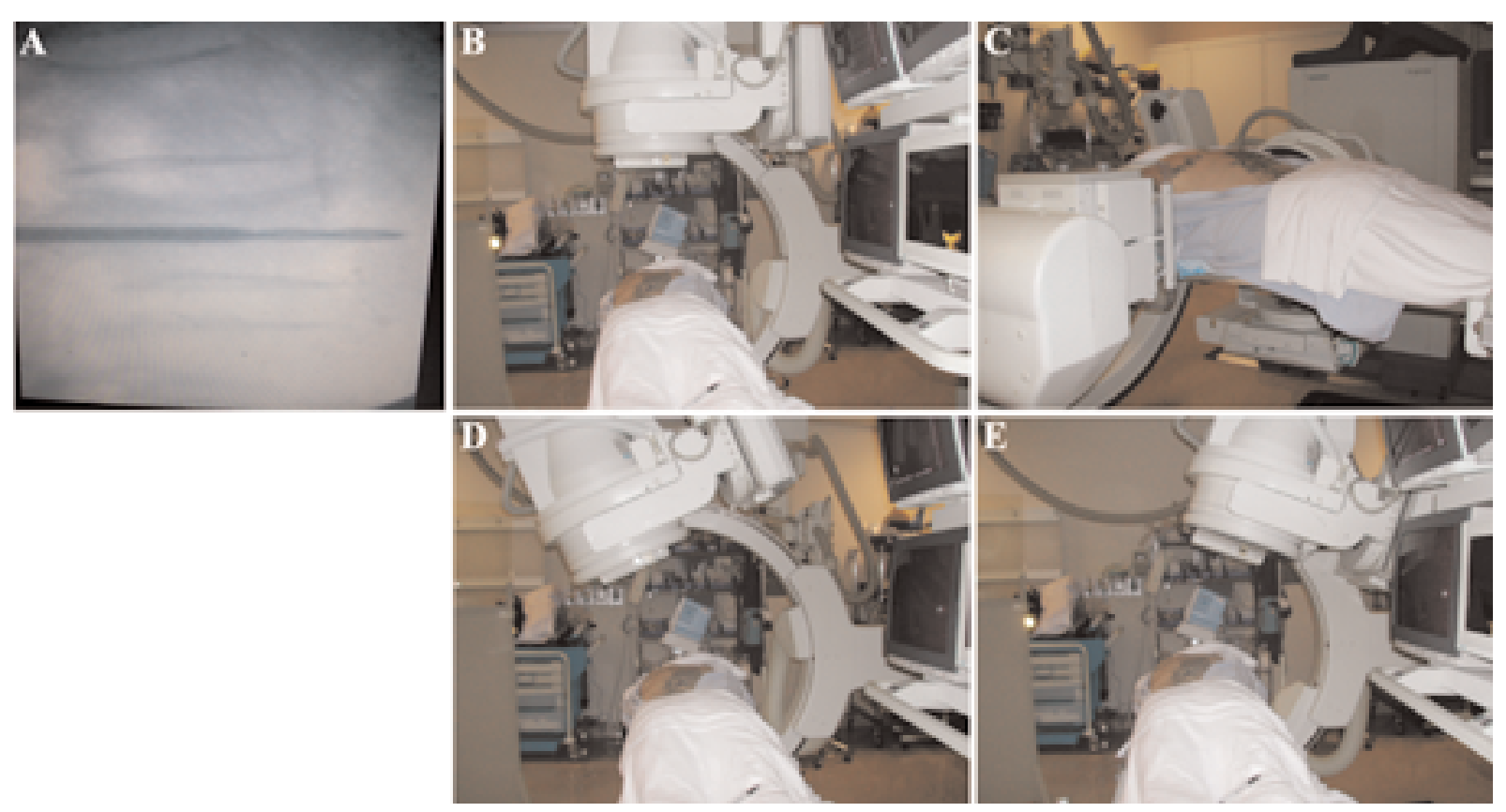

Fig. 4. A: Lateral fluoroscopic image. B-E: Fluoroscopic C-arm movements freely and rapidly rotating around a fixed target (the spine), which is placed in the center of rotation.

In Cases 5 and 6, there is breaching of the medial cortices of the pedicles. In Case 5, the needles pass through the lateral recesses on a course to injure the nerve roots of L-3 in the lateral recesses. In Case 6, the medial cortices of the pedicles are breached. This allows cement to flow easily into the spinal canal. One of the advantages of using a en face (looking down the bore of the needle) instead of a triangulation method to target locations in the pedicle is that one can more easily see a white zone of cancellous bone between the shaft of the needle and the inner cortex of the pedicles (Fig. 6B). This assures the operator that the needle is not too medial. In addition, starting at the upper outer aspects of the pedicle can help keep the needle from breaching the medial cortex of this structure and entering the spinal canal.

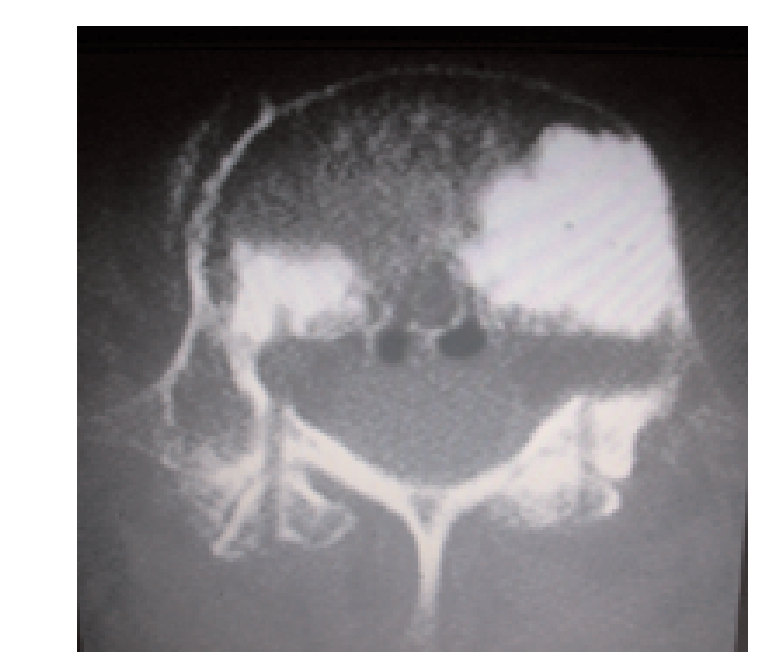

Fig. 5. Axial CT scan through VCF postvertebroplasty.

\section{Case 7}

After a vertebroplasty (Fig. 7A), the pain persists in this patient. Why?

The lateral view (Fig. 7B) demonstrates the problem in this case, in which needle placement is faulty. If the trajectory is not properly planned beforehand, the tips of the needles can enter the disc space above, breaching the superior endplates. This allows for leakage outside of the vertebra; also, in this case there is insufficient cementation in the vertebra to stabilize the fracture for pain control. It is important to preplan the needle trajectory so that the needles bisect the fracture lines (Fig. 7C). This can best be performed by projecting the trajectories on the lateral view prior to needle entry. Aligning the pedicles to superimpose them on the midsection of the VBs can also help. This is extremely important in the upper thoracic regions, where the pedicles meet the vertebrae at an angle.

\section{Case 8}

After vertebroplasty, this patient experienced worsening of pain, including a new pain along the left T6-8 intercostal spaces (Fig. 8A). Why?

Vertebroplasty at T-7 in this case involves faulty needle placement, in which the tip of the needle is not placed deeply enough. The tip ideally should be in the anterior quarter of the vertebra. Also, ideally the needle should pass from posterior to anterior with a slight anterior-tomedial angulation. In this case, because the needle position is too far posterior in the vertebrae (Fig. 8B), there is a greater cement flow posteriorly. Also, the operator has failed to realize that cement is extravasating posterior to the vertebrae. This indicates that a number of additional mistakes have been made, including failure to visualize the cement adequately, probably in part due to substandard fluoroscopy and also to inadequate opacification of 


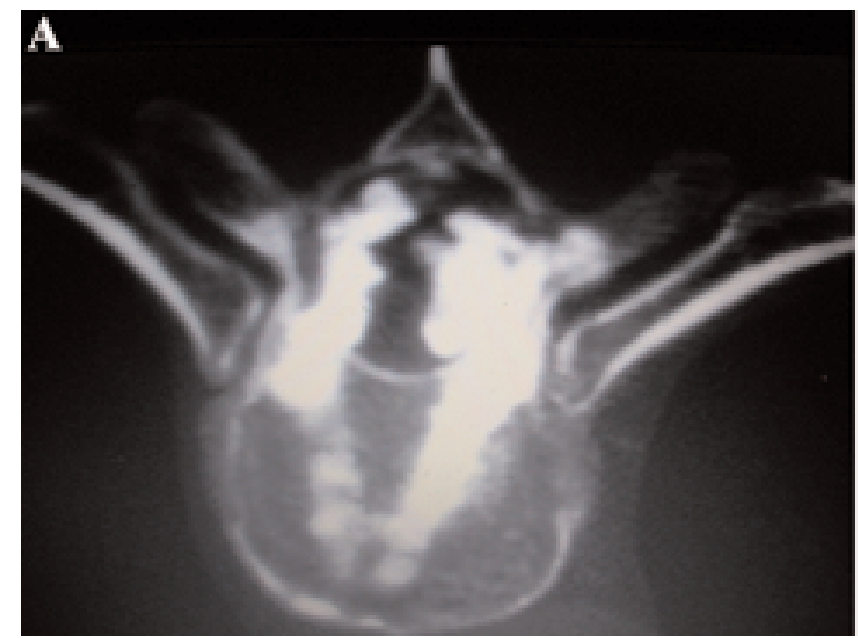

Fig. 6. A: Axial CT scan through VCF postvertebroplasty. dle entry for vertebroplasty.

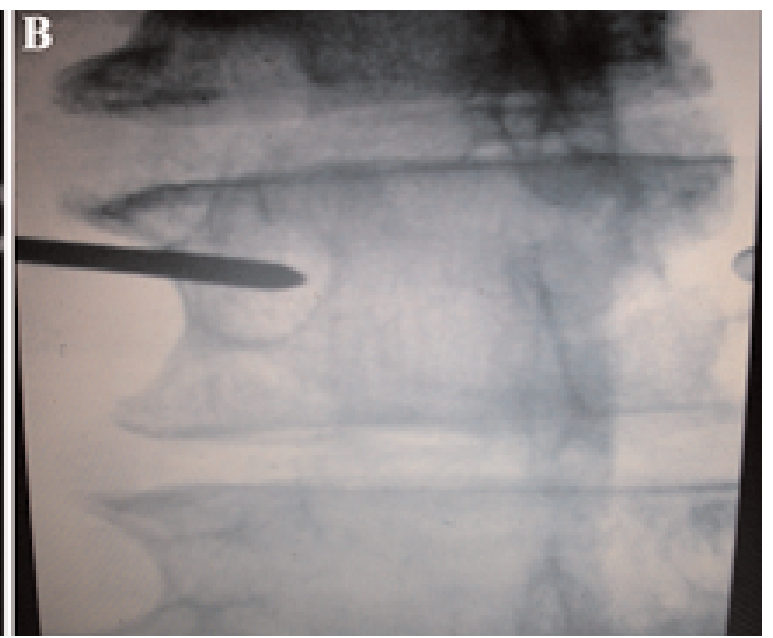

B: Oblique fluoroscopic image of pedicle during nee- the cement. This problem is further illustrated on the follow-up reconstructed CT scan (Fig. 8C). Here, cement can be seen to extrude well into the canal and the left T6-8 neuroforamina.

\section{Case 9}

In this hypothetical case, a diagram (Fig. 9A) is shown of the needle placement recommended for a vertebroplasty by a manufacturer of vertebroplasty equipment who promotes a single posterolateral entry with a large-bore needle system. What problems can this technique lead to?

Figure 9B is an MR image that correlates the center of the vertebra (arrowhead), which of course represents the center of the epidural venous plexus. This method could promote leakage directly back through epidural veins to the epidural venous plexus along the anterior aspect of the spinal canal. (Figure 9C is an example of cement extravasation along epidural veins into the spinal canal as a result of needle placement in the center of the vertebra rather than in the anterior quarter.) Also, the single posterolateral approach into the center of the vertebra carries the possibility of transecting the segmental artery or even injuring the exiting nerve root (Fig. 9D). Figure 9E demonstrates the course of the needle during a single posterolateral approach (arrow). It can be seen that the course of the nee- dle potentially endangers the nerve root and segmental artery.

The safest means of entry into the vertebrae is by way of the transpedicular approach (Fig. 6B). Nevertheless, for situations in which the pedicles are smaller than the needle, an extrapedicular (or parallel to the pedicle) technique can be used by driving the needle down through the costovertebral junction (Fig. 9F). This avoids the nerve root and segmental artery. This technique is frequently used in the upper thoracic region when the pedicles are very small. Figure 9G illustrates the costovertebral (parapedicular) entry as seen on CT scanning.

\section{Case 10}

After vertebroplasty for painful T-10 and T-11 VCFs, this patient does not experience significant pain relief (Fig. 10A). Why not?

On the lateral views (Fig. 10B and C), it is apparent that more than one error has been made. Not only has the operator failed to treat the correct levels (T-10 and T-11, not T10 and $\mathrm{T}-12$ ), but only half of the T-10 vertebra has been cemented. This is insufficient for stability.

A major advantage of using a bipedicular rather than a unipedicular approach is that with a bipedicular procedure, the filling of both sides of the vertebrae is more reli-
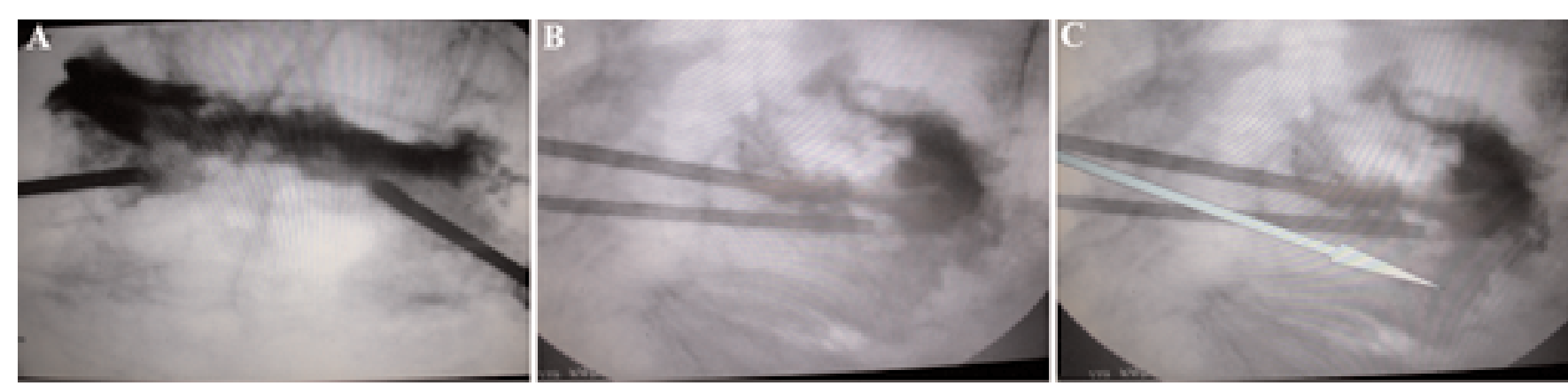

Fig. 7. A: Anteroposterior fluoroscopic image postvertebroplasty. B and C: Lateral fluoroscopic images postvertebroplasty. 

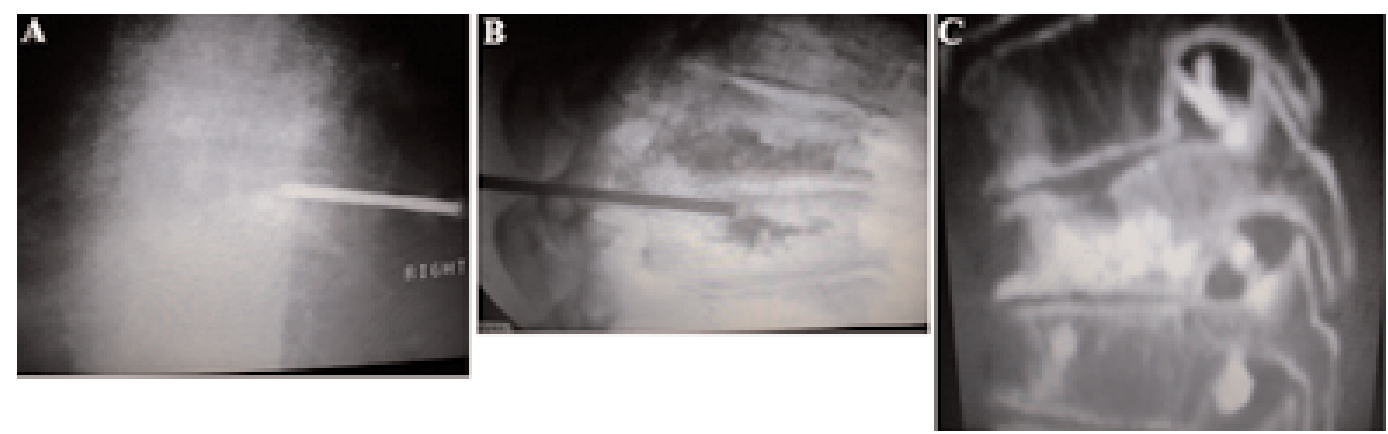

Fig. 8. A: Anteroposterior portable fluoroscopic image during vertebroplasty. B: Lateral portable fluoroscopic image during vertebroplasty. C: Sagittal CT scan of reconstruction through lumbar neuroforamina.

ably accomplished. If a cement leak occurs on one side, the injection can be stopped and resumed on the other side. This makes cement leaks less likely and the procedure is safer. Incidentally, for a unipedicular approach, it is important that the needle cross the midline to fill both sides of the vertebrae adequately.

In this case, the operator claims that he had trouble seeing the right-sided pedicles for T-10 and T-11. If that is the case, he should try to medialize the needles more, so that they cross the midline. If he chooses not to do that, he could reschedule the patient to be treated with the aid of high-quality digital fluoroscopy instead of low-quality portable analog fluoroscopy. Other techniques that might help include lining up the pedicles above and below the vertebra in which the pedicles are difficult to see, and rotating the fluoroscope back and forth often can be helpful in defining the outline of the pedicles. In the upper thoracic region, where the kyphosis may be extreme, sighting
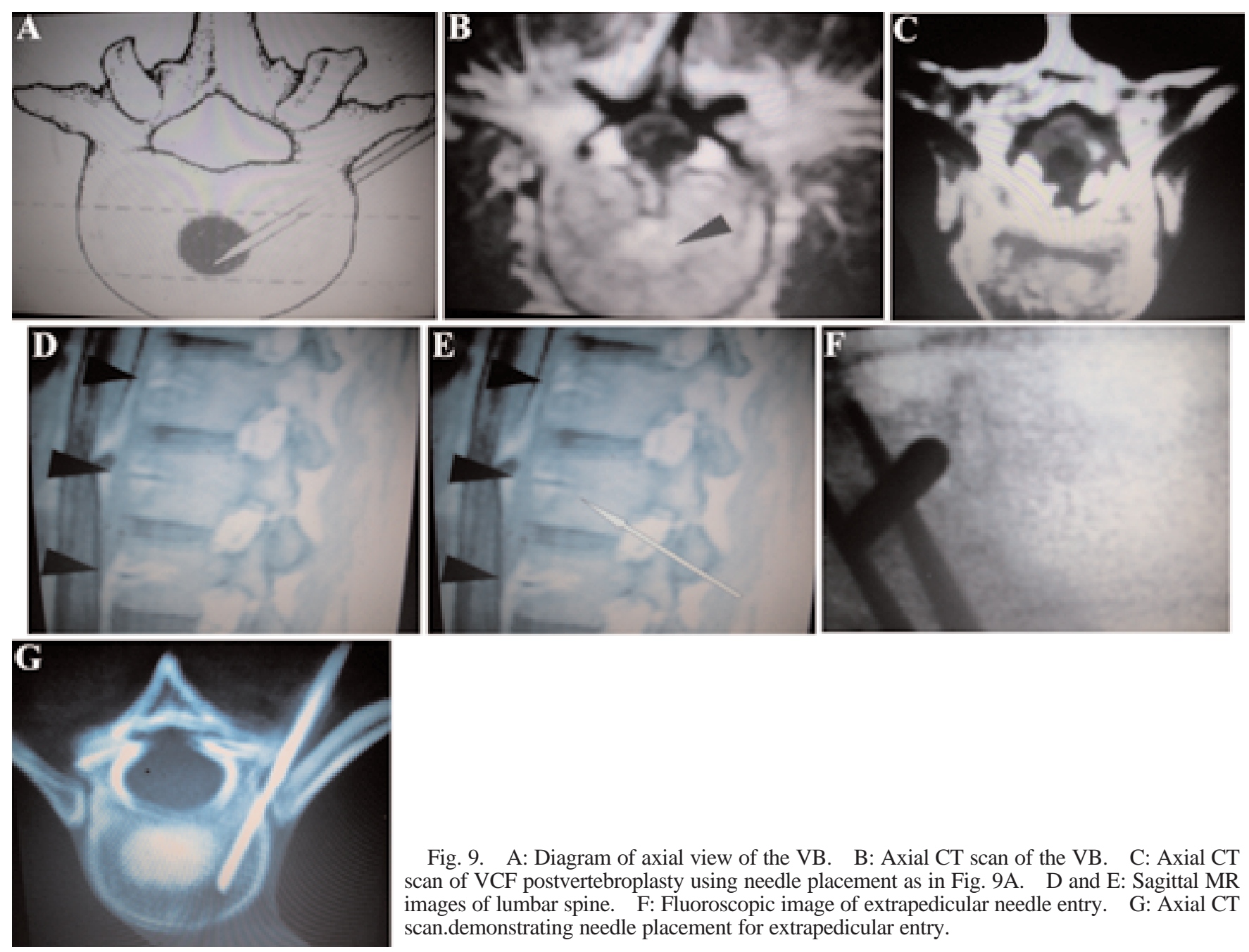

Fig. 9. A: Diagram of axial view of the VB. B: Axial CT scan of the VB. C: Axial CT scan of VCF postvertebroplasty using needle placement as in Fig. 9A. D and E: Sagittal MR images of lumbar spine. F: Fluoroscopic image of extrapedicular needle entry. G: Axial CT scan.demonstrating needle placement for extrapedicular entry. 

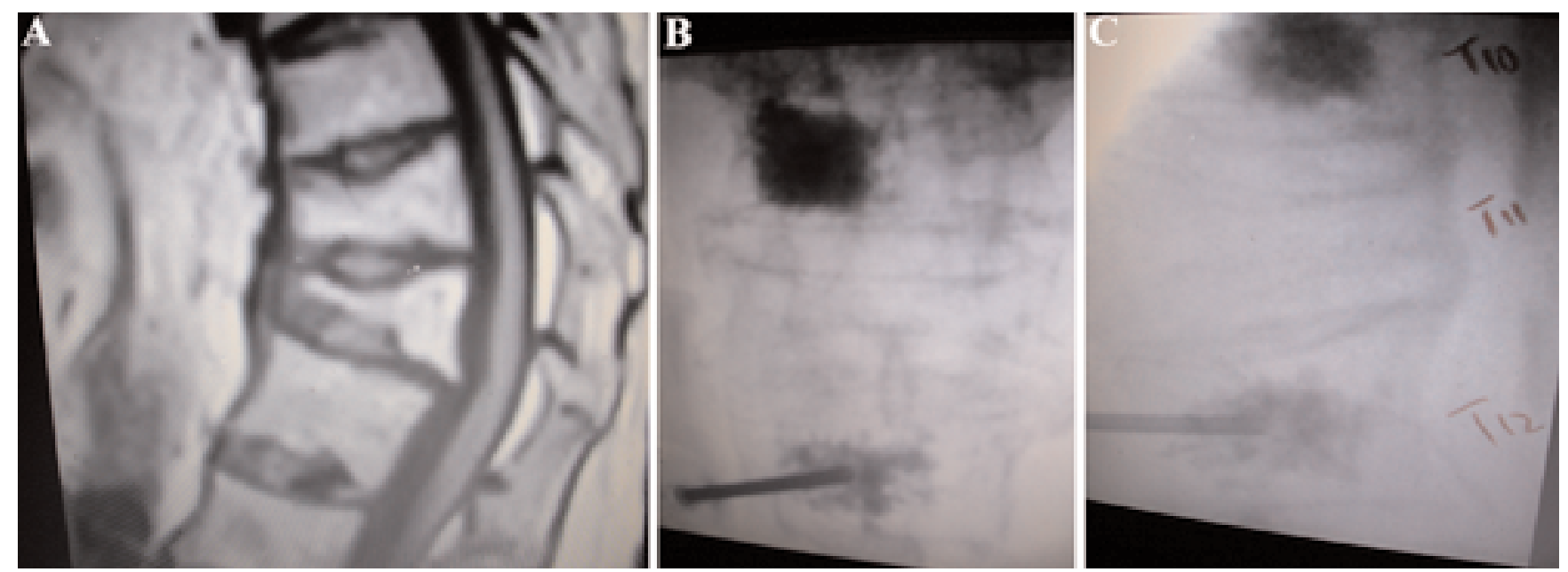

Fig. 10. A: Sagittal $\mathrm{T}_{1}$-weighted MR image of T-10 and T-11 VCFs. B: Anteroposterior analog portable fluoroscopic image. C: Lateral analog portable fluoroscopic image.

down the pedicles to superimpose them on the midportion of the target VB may clarify the trajectory. In addition, planning the trajectory of needle placement on the lateral view can be quite helpful in establishing the level of the starting point for the AP image. In the worst-case scenario, if the operator still has difficulty in adequately treating the target, he or she might choose to refer the case to a more experienced operator with superior fluoroscopy equipment.

\section{Case 11}

Kyphoplasty is performed in a patient after induction of general anesthesia. The patient awakes with paraplegia (Fig. 11A and B). Why?

A sign denoting that cement was injected is present, because the material is difficult to visualize and leakage occurred along epidural veins into the spinal canal. This confirms that there is a problem with adequate visualization. Because both vertebroplasty and kyphoplasty are imaging-guided procedures, adequate imaging is unquestionably essential; the operator really needs to use highquality digital fluoroscopy. In addition, cement opacification must be adequate. This requires the addition of sterile barium ( $30 \%$ by weight) to polymethyl methacrylate. In this case, because the operator has failed to visualize the cement he continues to inject more of it, thinking that he has not adequately filled the vertebra. This causes him to overfill greatly, spilling cement into the spinal canal. The cement leak is not detected until the patient awakes from general anesthesia, at which point paralysis is found in her lower extremities. A spinal decompression is performed, but it is already too late.

In addition to inadequate fluoroscopy and inadequate opacification, the operator is probably injecting cement in too liquid (runny) a state, so that it flows very easily from the vertebrae into the spinal canal. If the operator had waited longer after mixing the cement to allow it to reach a very viscous, puttylike state, the material might have been more readily contained in the vertebra. In addition, the delay and inability of the operator to assess the patient postoperatively is exacerbated by the long recovery time from general anesthesia. This type of anesthesia not only prolongs recovery time but prevents monitoring of neurological changes during the actual procedure. Figure $11 \mathrm{C}$ is an example of sharp visualization by using digital fluoroscopy and high-opacity material. The instant a small
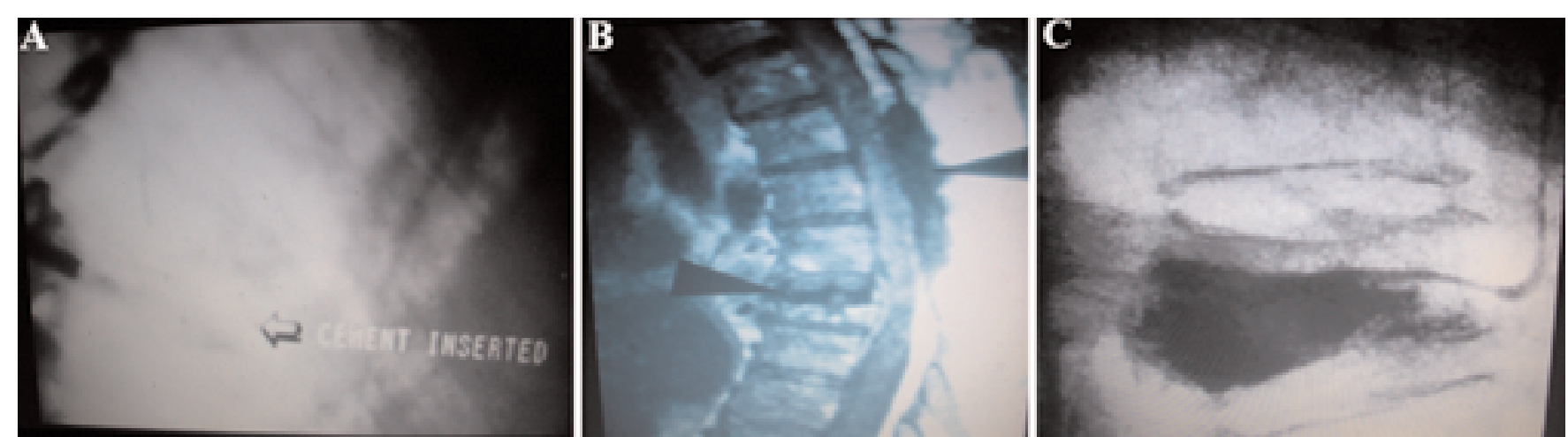

Fig. 11. A: Lateral analog portable fluoroscopic image. B: Sagittal $\mathrm{T}_{1}$-weighted MR image. $\quad$ C: Lateral digital fluoroscopic image 


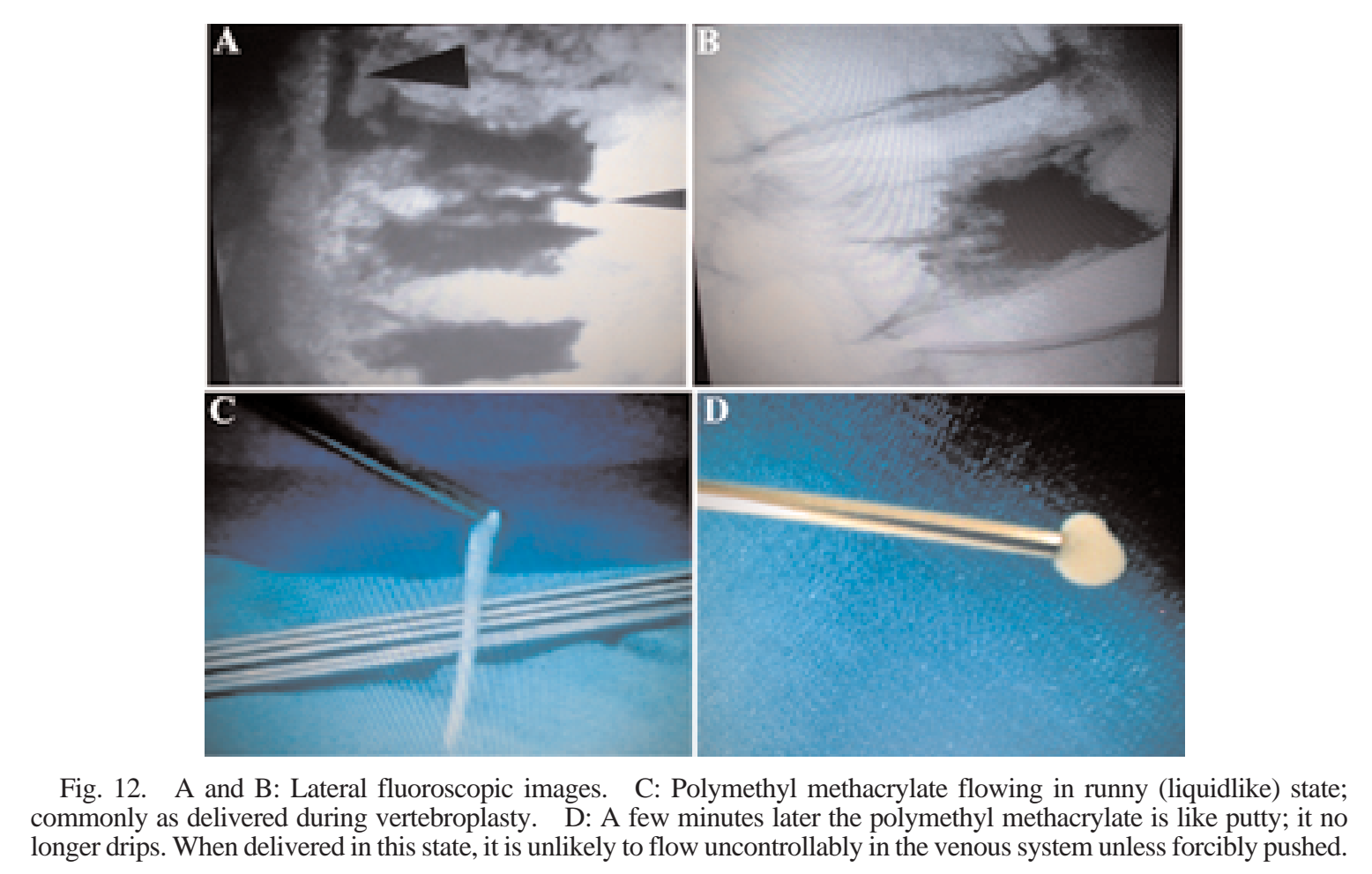

leak is seen (anteriorly in this case), the operator should stop injecting cement. Wait! Let the cement harden before resuming.

\section{Case 12}

What are the problems here (Fig. 12A)? Why did they occur?

In this case, there is leakage of barium-opacified cement into the epidural space as well as into the adjacent disc space. This is caused by overfilling of the vertebra. Figure 12B demonstrates adequate filling of the vertebra from the anterior to the posterior portion, with filling of the anterior three-fourths of the vertebra (the stress zone is anterior). If one sees a small amount of leakage along the epidural veins, then it is time to stop the injection. Belkoff, et al., ${ }^{1}$ suggest that as little as $2.5 \mathrm{~cm}^{3}$ of cement in the upper thoracic and $4 \mathrm{~cm}^{3}$ in the thoracolumbar/lumbar region are usually adequate to ameliorate pain and strengthen the vertebrae to their prefracture state.

Figure 12C depicts cement flowing in a liquid state, whereas Fig. 12D depicts cement a few minutes later in a highly viscous, doughy, puttylike state. In this viscous state, if the cement gets into a vein, it will not flow as easily as in liquid form unless it is pushed. This permits more control and a greater margin of safety.

\section{OTHER SAFETY CONSIDERATIONS}

\section{What's the Problem Here?}

Some dangers of vertebroplasty or kyphoplasty may involve the operator instead of the patient (Table 2). One mark of an inexperienced operator is fluoroscopy of his or her hands (Fig. 13A). Avoid radiation exposure of body parts. Manipulate needles with forceps (Fig. 13B) and
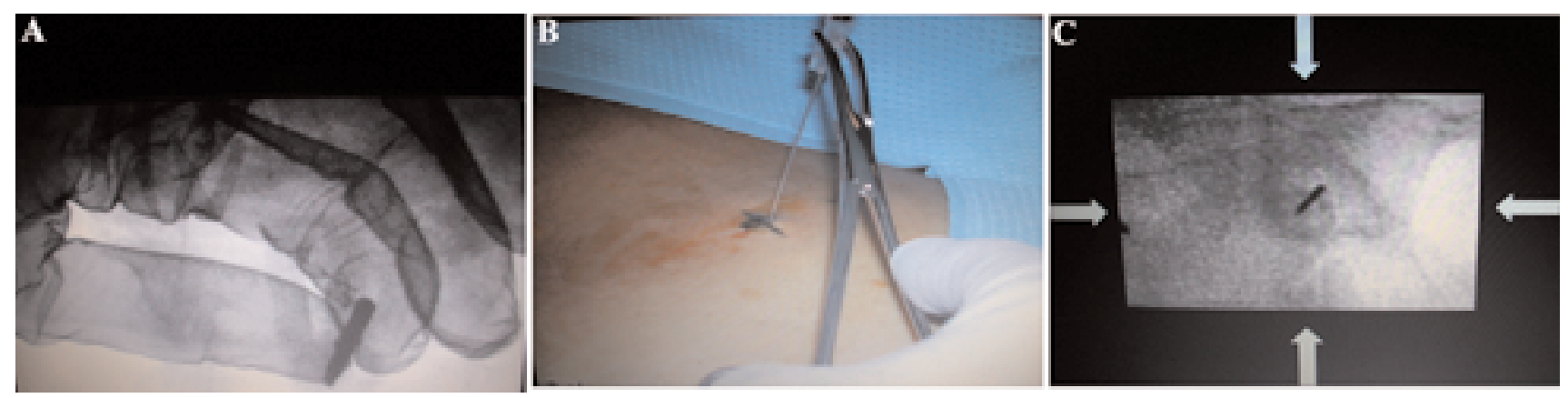

Fig. 13. A: A common mistake: exposing hands to radiation unnecessarily. B: Use of forceps to avoid radiation exposure. C: Narrowing collimators helps reduce exposure and improves image quality. 

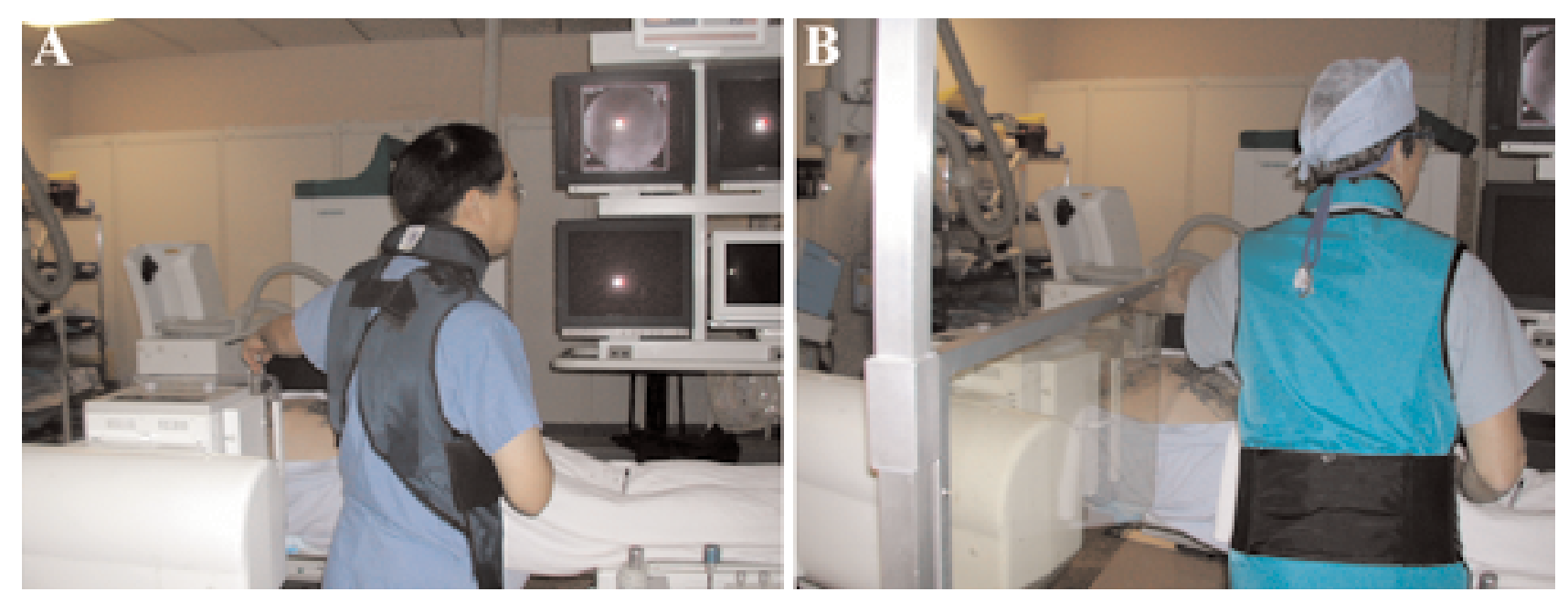

Fig. 14. A: A common mistake: radiation exposure to an unprotected back when the fluoroscopic tube is in the lateral position. B: One solution is to interpose a lead-glass shield between the x-ray tube and the operator and wear a wrap-around lead apron that protects front and back.

with the intermittent controlled fluoroscopy. Tight collimation (Fig. 13C) not only permits the operator to work closer to the operating field without direct radiation exposure, but this also sharpens the fluoroscopic image because it reduces scatter.

\section{What's Wrong With This Picture?}

In this case (Fig. 14A), the radiation is directed away from the operator; however, he has his back turned to the tube casing and there is no lead shielding to protect him from scatter radiation emanating from this part of the apparatus. In addition, he is standing very close to the tube casing, and this can represent a substantial radiation exposure to the operator.

Figure 14B demonstrates a more protected setup, with a lead glass shield interposed between the operator and the $\mathrm{x}$-ray tube. Also, the operator is wearing a wraparound lead apron, which protects not only his front but also his back and sides. Other safety aids would include lead gog- gles, lead gloves, thyroid shields, forceps, and audible dosimeters.

Other dangers to the operator quite often involve needles. Danger lurks when leaving needles lying randomly in the operating field (Fig. 15A). As shown in Fig. 15B, transferring needles to assistants can be done irresponsibly. One should always handle and transfer needles as if they are firearms (Fig. 15C). Avoid recapping needles (Fig. 15D), but if you must recap, lay the cap down when inserting the needle (Fig. 15E). As shown in Fig. 15F, needles should be stored properly to avoid inadvertent injury. When the procedure is complete, needles should also be disposed of properly (Fig. 15G) so that ancillary personnel are not inadvertently injured.

\section{CONCLUSIONS}

Vertebroplasty and kyphoplasty are minimally invasive imaging-guided procedures that can be performed at very

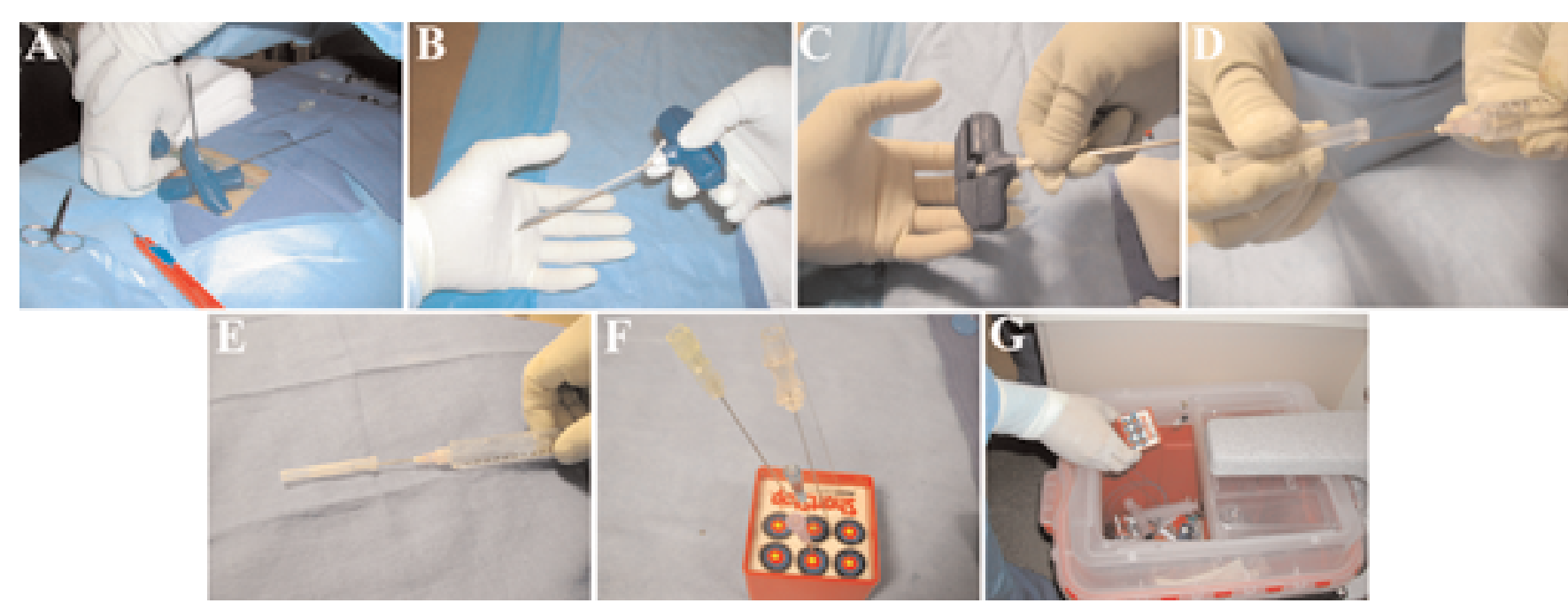

Fig. 15. A-D: Common mistakes with needle handling. E-G: Common techniques for safe needle handling. 
low risk if an awareness of the dangers is kept in mind. High-quality fluoroscopy, a thorough understanding of imaging anatomy, and the use of adequately opacified and viscous cement are further essentials.

\section{Disclosure}

Dr. Wong has been on the training faculty for the following companies: Kyphon, Spinal Specialties, Stryker, and Arthrocare. Dr. Mathis has been a consultant for Stryker and Kyphon, and he has been on the training faculty for Stryker.

\section{References}

1. Belkoff SM, Mathis JM, Jasper LE, et al: The biomechanics of vertebroplasty. The effect of cement volume on mechanical behavior. Spine 26:1537-1541, 2001

2. Chiras J, Deramond H: Complications des vertébroplasties, in Saillants G, Laville C (eds): Echecs et Complication de la Chirurgie du Rachis. Chirurgie de Reprise. Paris: Sauramps Medical, 1995, pp 149-153

3. Jensen ME, Evans AJ, Mathis MJ, et al: Percutaneous polymethylmethacrylate vertebroplasty in the treatment of osteoporotic vertebral body compression fractures: technical aspects. AJNR 18: 1897-1904, 1997

4. Mathis JM: Percutaneous vertebroplasty: complication avoidance and technique optimization. AJNR 24:1697-1706, 2003
5. Mathis JM, Barr JD, Belkoff SM, et al: Percutaneous vertebroplasty: a developing standard of care for vertebral compression fractures. AJNR 22:373-381, 2001

6. Mathis JM, Deramond H, Belkoff SM: Percutaneous Vertebroplasty. New York: Springer, 2002, p 200

7. Phillips FM, Wetzel T, Lieberman I, et al: An In vivo comparison of the potential for extravertebral cement leak after vertebroplasty and kyphoplasty. Spine 27:2173-2179, 2002

8. Theodorou DJ, Wong WH, Duncan TD, et al: Percutaneous balloon kyphoplasty: a novel technique for reducing pain and spinal deformity associated with osteoporotic vertebral compression fractures. Radiology 217 (Suppl):511, 2000 (Abstract)

9. Weill A, Chiras J, Simon JM, et al: Spinal metastases: indications for and results of percutaneous injection of acrylic surgical cement. Radiology 199:241-247, 1996

10. Wong W, Mathis J: Is intraosseous venography a significant safety measure in the performance of vertebroplasty. $\mathbf{J}$ Vasc Interv Radiol 13:137-138, 2002

11. Wong W, Reiley MA, Garfin S: Vertebroplasty/kyphoplasty. J Women's Imaging 2:117-124, 2000

Manuscript received January 14, 2005.

Accepted in final form February 14, 2005.

Address reprint requests to: Wade Wong, D.O., F.A.C.R., Department of Radiology \#7756, University of California San Diego Medical Center, 9300 Campus Point Drive, La Jolla, California 92037. email: cott@ucsd.edu. 